\title{
Clinical associations and time of onset of cerebral white matter damage in very preterm babies
}

National Perinatal Epidemiology Unit, Radcliffe Infirmary NHS Trust,

Oxford

D J Murphy

A Johnson

Department of Neuropathology MV Squier

Department of Paediatrics, John Radcliffe Hospital, Oxford

P L Hope

Women's Centre, John Radcliffe Hospital Susan Sellers

Correspondence to: Dr DJ Murphy, 22 Manor Park, Redland,

Bristol BS6 7HH.

- Crown copyright 1996

Accepted 10 April 1996

\author{
Deirdre J Murphy, Marian V Squier, Peter L Hope, Susan Sellers, Ann Johnson
}

\begin{abstract}
Neuropathological examinations were carried out at necropsy on 83 very preterm babies who died during their first hospital admission. Forty seven (57\%) babies had evidence of cerebral damage -39 with ischaemic white matter damage. The time of onset of ischaemic lesions was thought to be prenatal in 12 cases (31\%) and postnatal in a further 12 $(31 \%)$. The exact timing of damage could not be determined in $15(38 \%)$ cases. Maternal and neonatal case notes were reviewed to ascertain clinical associations of ischaemic white matter damage. There were no clear associations between adverse clinical factors and prenatal ischaemic white matter damage. In contrast, pre-eclampsia, intrauterine growth retardation, and delivery without labour were associated with postnata,l damage as were neonatal sepsis, necrotising enterocolitis, and seizures.

The absence of a clear association between the timing of adverse clinical factors and the timing of ischaemic cerebral damage suggests that cerebral damage in very preterm babies may result from a sequence of events rather than one specific insult.

(Arch Dis Child 1996;75:F27-F32)
\end{abstract}

Keywords: cerebral white matter damage, prenatal ischaemia, very preterm, neuroimaging.

During the $1980 \mathrm{~s}$, there was evidence of a sharp increase in the rate of cerebral palsy among babies with a birthweight of less than $1500 \mathrm{~g}$. This is thought to reflect an increase in their survival. ${ }^{1}$ The increased cerebral palsy rate might reflect the survival of babies who have sustained a cerebral insult in utero and were born preterm, or alternatively, the survival of immature babies who are particularly vulnerable to haemorrhage and ischaemia in the intrapartum and neonatal period. ${ }^{2} \mathrm{~A}$ third possibility is that cerebral palsy in very preterm babies is the endpoint of a continuum of adverse events which occur throughout the period when the developing white matter is particularly vulnerable to injury-that is, 26-34 weeks of gestation. These adverse events might occur before, during, or after birth.

The available ways of assessing the time of brain injury are limited. Because of the well described association between cystic lesions detected on cerebral ultrasonography and subsequent cerebral palsy, ${ }^{3-7}$ neuroimaging around or after the time of birth provides one way of estimating when cerebral palsy arises. Occasionally, antenatal neuroimaging can demonstrate severe and well established cystic white matter lesions that have arisen before birth. ${ }^{8}$ More frequently, lesions are first detected by neuroimaging of the infant brain after birth. Some of the lesions detected in this way have clearly been present before birth, ${ }^{8-10}$ but more frequently, the lesions are first seen days or weeks after birth. The early changes following an ischaemic insult, however, may not be detected by current methods of cranial ultrasound, and this places a severe constraint on this method as a means of timing the onset of brain lesions with any precision.

An alternative approach is to study the time of onset of cerebral lesions seen on neuropathological study in babies who die. Ischaemic white matter lesions, cerebral malformations, hydrocephalus following intraventricular haemorrhage, and cerebral infection can all be clearly defined at necropsy, and when they occur, can be estimated with reasonable certainty. Babies with any of these conditions, had they survived, might have shown signs of cerebral palsy. Indeed, study of the brains of children with cerebral palsy who die shows that a proportion have white matter damage of varying severity which could plausibly be the end result of the ischaemic white matter lesions seen on perinatal neuropathological study (unpublished data). In a previous neuropathological study of the prevalence and characteristics of cerebral lesions in babies of all gestational ages who either die in utero or early in the neonatal period, the commonest lesion was ischaemic white matter injury. ${ }^{11}$ A proportion of these ischaemic lesions occurred prenatally. In view of the likely association between these ischaemic white matter lesions in babies who die and the lesions which are precursors of cerebral palsy in babies who survive, we studied the brains of very preterm singleton babies who die during their first hospital admission. The following questions were addressed. What is the prevalence of white matter lesions in very preterm babies who die and the clinical factors associated with the lesions? Do the clinical factors, which are associated with lesions of prenatal origin, differ from those associated with lesions of postnatal origin? We also wanted to explore the relation between 
Table 1 Approximate timing of cellular responses to injury in fetal brain

\begin{tabular}{ll}
\hline Reactive astrocytosis & 12 hours-4 days \\
Fibrillary gliosis & 6 days \\
Microglial proliferation & 3 hours-3 days \\
Macrophage infiltration & $4-6$ days \\
Neuronal kayorrhexis & $12-48$ hours \\
Capillary endothelial reduplication & 5 days \\
Cyst formation & $10-42$ days
\end{tabular}

brain lesions seen on postnatal cerebral ultrasonography and lesions seen at necropsy.

\section{Methods}

Eighty three singleton babies live born between 1984 and 1994 at less than 32 completed weeks of gestation, who died during their first hospital admission and who had a neuropathological examination at necropsy, were included in the study. The mothers of $59(71 \%)$ of them were residents of one health district; the remainder were referred by obstetricians and paediatricians from other health districts. It was, therefore, a highly selected series.

Neuropathological examination was performed by a single observer (MVS). The presence of ischaemic white matter damage, cerebral malformation, hydrocephalus follow-

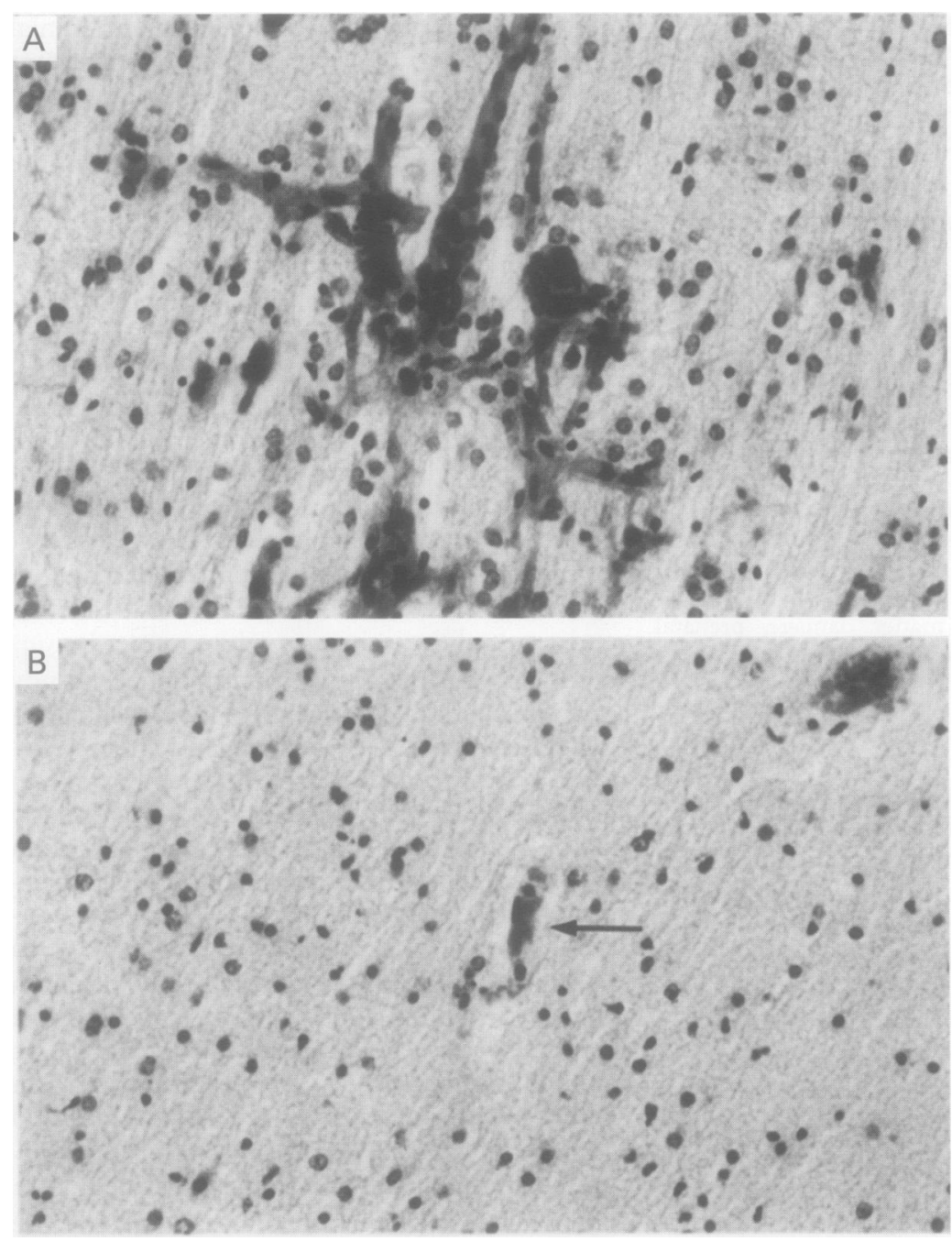

Figure 1 White matter from a 35 week old infant who died 20 minutes after birth with evidence of periventricular leucomalacia. Away from the macroscopically evident cysts, the white matter showed diffuse ischaemic damage with increased numbers of glial cells and macrophages and substantial capillary proliferation. (B) Fetus at 32 weeks old with no evidence of ischaemia. Note the normal cellularity of the white matter and normal capillary (arrow). ing extensive intraventricular haemorrhage, or cerebral infection was noted. The diagnosis of white matter ischaemic damage was based on finding evidence of cerebral infarction; in the more severe and long standing cases this was readily recognised as the changes of periventricular leucomalacia. Four criteria were used to establish a diagnosis of diffuse, mild, or early ischaemic white matter damage. These criteria were reactive astrocytosis, macrophage infiltration, karyorrhexis, and endothelial swelling or reduplication. Cerebral infection included ventriculitis and meningitis with or without coexistent ischaemic damage. Lesions were dated by assessment of a sequence of cellular reactions which occur in the weeks following injury. The timing of this sequence of events is outlined in table 1 and figs 1 and 2 , and is based on personal observations and data derived from published accounts of fetal neuropathology. ${ }^{12}$ The age of a lesion in relation to birth was used to identify pre- or postnatal injury. In some cases, usually those with long survival, the sequence of cellular reactions had ceased and timing was not possible except where a specific associated malformation could be identified, indicating an onset early in development.

The obstetric notes of mothers included in the study were obtained and reviewed by a researcher (DJM) who was blind to the necropsy findings. Gestational age was estimated by using a combination of menstrual dates and an ultrasound scan performed before 20 weeks' gestation. The scan date was preferred if the menstrual date was uncertain or there was a discrepancy of more than 14 days between the menstrual date and the scan estimate. A detailed data set was completed encompassing 130 variables, including demographic data, maternal medical history, family history, obstetric history and history of the current pregnancy. Pre-eclampsia, antepartum haemorrhage, prolonged rupture of the membranes, chorioamnionitis and other maternal infection were of particular interest and were described in detail in terms of diagnosis, onset, duration and management (definitions in Appendix 1). The occurrence of labour and the mode of delivery were recorded, as were characteristics of the infants at birth, including Apgar scores and umbilical cord blood assays. Postnatal complications of interest included prolonged ventilation, respiratory disease, hypotension, patent ductus arteriosus, sepsis, necrotising enterocolitis and neonatal seizures (definitions in Appendix 2). These data were extracted from the neonatal notes by the same observer (DJM).

Serial postnatal cerebral ultrasound examinations had already been recorded and stored on videotape. Ultrasound scanners were the ATL 300C (Advanced Technical Laboratories), until 1988, and the UM4 (ATL) thereafter with 7.5 MHZ transducer heads. For retrospective review, four recordings were selected and reviewed for each baby. These were taken from the first 24 hours after birth, between 25 and 72 hours, between 5 and 9 days, and at about 6 weeks. The findings were described 
Table 2 Characteristics of subjects with and without ischaemic white matter damage

\begin{tabular}{|c|c|c|}
\hline Characteristics & All lesions $(n=39)$ & No lesions $(n=36)$ \\
\hline \multicolumn{3}{|l|}{ Gestational age (weeks): } \\
\hline Median & 27 & 26 \\
\hline (Range) & $(24-32)$ & $(22-32)$ \\
\hline \multicolumn{3}{|l|}{ Birthweight (g): } \\
\hline Median & 1068 & 940 \\
\hline (Range) & $(425-3685)$ & $(390-2553)$ \\
\hline Sex (male n (\%)) & $16(41)$ & $22(61)$ \\
\hline Congenital abnormality (n (\%)) & $3(8)$ & $5(14)$ \\
\hline Small for gestational age (n (\%)) & $12(31)$ & $5(14)$ \\
\hline \multicolumn{3}{|l|}{ Age at death in days: } \\
\hline $\begin{array}{l}\text { Mean (SD) } \\
\text { (Range) }\end{array}$ & $\begin{array}{l}14(20) \\
\quad(1-94)\end{array}$ & $\begin{array}{l}20(49) \\
\quad(1-240)\end{array}$ \\
\hline Apgar score at 1 minute $\leqslant 3(\mathrm{n}(\%))$ & $21(54)$ & $20(56)$ \\
\hline Apgar score at 5 munutes $\leqslant 5$ & $8(21)$ & $14(39)$ \\
\hline $\mathrm{pH}$ of umibilical artery $\leqslant 7.10(\mathrm{n}(\%))$ & $4 / 34(12)$ & $3 / 34(9)$ \\
\hline $\begin{array}{l}\text { Base excess of umbilical artery } \geqslant 12.0 \\
(n(\%))\end{array}$ & $6 / 34(18)$ & $4 / 34(12)$ \\
\hline
\end{tabular}

Table 3 Antenatal, intrapartum, and neonatal characteristics for babies with and without ischaemic white matter lesions expressed as number (\%) and odds ratio OR (95\% confidence interval)

\begin{tabular}{lcccc}
\hline Variable & $\begin{array}{c}\text { All lesions } \\
(n=39)\end{array}$ & $\begin{array}{c}\text { No lesion } \\
(n=36)\end{array}$ & OR (95\% CI) & $\begin{array}{l}P \\
\text { value }\end{array}$ \\
\hline Antepartum haemorrhage & $12(31)$ & $10(28)$ & $1.2(0.4-3.1)$ & \\
Chorioamnionitis & $5(13)$ & $8(22)$ & $0.5(0.2-1.8)$ & \\
Any maternal infection & $7(18)$ & $8(22)$ & $0.8(0.3-2.4)$ & \\
Pre-eclampsia & $8(21)$ & $3(8)$ & $2.8(0.7-11.7)$ & $1.5(0.5-4.7)$ \\
Intrauterine growth retardation & $9(23)$ & $6(17)$ & $0.5(0.2-1.1)$ & \\
Vaginal vertex delivery & $13(33)$ & $19(53)$ & $0.7(0.2-2.9)$ & \\
Vaginal breech delivery & $4(10)$ & $5(14)$ & $4.8(1.6-14.0)$ & 0.007 \\
Caesarean section, no labour & $19(49)$ & $6(17)$ & $0.4(0.1-1.8)$ & \\
Caesarean section, in labour & $3(8)$ & $6(17)$ & $1.0(0.4-2.9)$ & \\
Pneumothorax & $10(26)$ & $9(25)$ & $1.1(0.4-3.3)$ & \\
Hypotension & $31(79)$ & $28(78)$ & $0.9(0.3-2.4)$ & \\
Patent ductus arteriosus & $11(28)$ & $11(31)$ & $1.3(0.5-3.3)$ & \\
Sepsis & $14(36)$ & $11(31)$ & $12.1(1.5-99)$ & 0.01 \\
Necrotising enterocolitis & $10(26)$ & $1(3)$ & $9.5(2.0-46)$ & 0.003 \\
Neonatal seizures & $14(36)$ & $2(6)$ & & \\
\hline
\end{tabular}

${ }^{\star} \chi^{2}$ test with Yates's correction, or Fisher's exact test where an expected value is less than 5 .

Table 4 Antenatal, intrapartum, and neonatal characteristics for babies with prenatal ischaemic lesions and with no ischaemic white matter lesions

\begin{tabular}{llll}
\hline Variable & $\begin{array}{l}\text { Prenatal lesions } \\
(n=12)\end{array}$ & $\begin{array}{c}\text { No lesions } \\
(n=36)\end{array}$ & OR (95\% CI) \\
\hline Antepartum haemorrhage & $5(42)$ & $10(28)$ & $1.9(0.5-7.2)$ \\
Chorioamnionitis & $1(8)$ & $8(22)$ & $0.3(0.04-2.9)$ \\
Any maternal infection & $2(17)$ & $8(22)$ & $0.7(0.1-3.9)$ \\
Pre-eclampsia & $1(8)$ & $3(8)$ & $1.0(0.1-10.6)$ \\
Intrauterine growth retardation & $3(25)$ & $6(17)$ & $1.7(0.3-8.0)$ \\
Vaginal vertex delivery & $6(50)$ & $19(53)$ & $0.9(0.2-3.3)$ \\
Vaginal breech delivery & $1(8)$ & $5(14)$ & $0.6(0.1-5.4)$ \\
Caesarean section, no labour & $4(33)$ & $6(17)$ & $2.6(0.6-11.4)$ \\
Caesarean section, in labour & $1(8)$ & $6(17)$ & $0.5(0.1-4.2)$ \\
Pneumothorax & $3(25)$ & $9(25)$ & $1.0(0.2-4.5)$ \\
Hypotension & $8(67)$ & $28(78)$ & $0.6(0.1-2.4)$ \\
Patent ductus arteriosus & $1(8)$ & $11(31)$ & $0.2(0.02-1.8)$ \\
Sepsis & 0 & $11(31)$ & \\
Necrotising enterocolitis & 0 & $1(3)$ & \\
Neonatal seizures & $3(25)$ & $2(6)$ & $5.7(0.8-39.2)$ \\
\hline
\end{tabular}

using a modified version of a classification that has been used before in a trial of surfactant (OSIRIS). ${ }^{13}$ The right and left cerebral hemispheres were described separately in terms of germinal layer/intraventricular haemorrhage, parenchymal echodensity, ventricular dilatation and parenchymal cysts. Moderate ventricular dilatation was assigned where the ventricular index was above the 97th centile, and hydrocephalus when the dilatation was more than $4 \mathrm{~mm}$ above the 97 th centile using a centile chart from Levene et al (1981). Parenchymal cyst was an umbrella term used for any parenchymal echolucency suggestive of a cavity. All of the recordings were reviewed by the same observer $(\mathrm{PH})$ who was blind to the identity of the child and the necropsy findings.

The results of all comparisons are presented as odds ratios and $95 \%$ confidence intervals. The two tailed $\chi^{2}$ test with Yates's correction was used to test for significant differences in proportions between those with and without lesions. Fisher's exact test was preferred where numbers were small. For continuous variables, Student's $t$ test was used to identify differences between mean values. A $P$ value of less than 0.05 was regarded as significant.

\section{Results}

Of 574 liveborn singletons admitted to the John Radcliffe special care baby unit, Oxford, between 1984 and 1994, at less than 32 completed weeks of gestation, 129 (24\%) died and of these $83(64 \%)$ had a neuropathological examination at necropsy. In 36 there were no cerebral lesions. Among the 47 cases with cerebral damage $(57 \%)$, two had congenital cerebral malformations, three ventriculitis/ meningitis, and three hydrocephalus following intraventricular haemorrhage. These eight cases are excluded from further analyses. Of the 39 babies with ischaemic white matter damage, $12(31 \%)$ were thought to be prenatal and $12(31 \%)$ postnatal in origin, and a further $15(38 \%)$ were of uncertain origin. Among those cases of severe ischaemic white matter damage, 16 had large cystic lesions and seven had gross cerebral atrophy.

Baseline characteristics at birth were similar for those with and without ischaemic white matter damage, and although there was a higher proportion of males and congenital abnormalities among babies with no lesions, and a higher proportion of small for gestational age babies among those with lesions, these differences were not significant at the $5 \%$ level (table 2). However, babies with ischaemic lesions died earlier than babies with no lesions $(P=0.005)$.

There were no significant differences in the numbers of adverse antenatal events when comparing those with and without ischaemic lesions (table 3). Of the intrapartum factors, however, caesarean section with no labour was associated with an increased risk of ischaemic cerebral damage: odds ratio $4.8(1.6-14.0)$. Of the neonatal factors studied, necrotising enterocolitis (odds ratio $12.1(1.5-99)$ ) and neonatal seizures (odds ratio $9.5(2.0-46)$ ) were associated with a significant increased risk of ischaemic damage. On comparing the clinical characteristics of those with prenatal ischaemic lesions and those without, antepartum haemorrhage, caesarean section without labour, and neonatal seizures occurred more frequently among those with lesions, but these differences were not significant at the $5 \%$ level (table 4). There was no association between pre-eclampsia and prenatal ischaemic damage. In contrast, pre-eclampsia was associated with a significantly increased risk of postnatal ischaemic cerebral damage: odds ratio $11.0(2.1-57)$ (table $5)$. There was also an increased risk of postnatal ischaemic white matter damage in association with intrauterine growth retardation (odds ratio 
Table 5 Antenatal, intrapartum, and neonatal characteristics for babies with postnatal ischaemic lesions and with no ischaemic white matter lesions

\begin{tabular}{|c|c|c|c|c|}
\hline Variable & $\begin{array}{l}\text { Postnatal lesions } \\
(n=12)\end{array}$ & $\begin{array}{l}\text { No lesions } \\
(n=36)\end{array}$ & OR $(95 \% C I)$ & $P$ value ${ }^{\star}$ \\
\hline Antepartum haemorrhage & $1(8)$ & $10(28)$ & $0.2(0.03-2.1)$ & \\
\hline Chorioamnionitis & $2(17)$ & $8(22)$ & $0.7(0.1-3.9)$ & \\
\hline Any maternal infection & $1(8)$ & $8(22)$ & $0.3(0.04-2.9)$ & \\
\hline Pre-eclampsia & $6(50)$ & $3(8)$ & $11.0(2.1-57)$ & 0.006 \\
\hline $\begin{array}{l}\text { Intrauterine growth } \\
\text { retardation }\end{array}$ & $6(50)$ & $6(17)$ & $5.0(1.2-21)$ & 0.04 \\
\hline Vaginal vertex delivery & $2(17)$ & $19(53)$ & $0.2(0.03-0.9)$ & \\
\hline Vaginal breech delivery & 0 & 5 (14) & & \\
\hline $\begin{array}{l}\text { Caesarean section, no } \\
\text { labour }\end{array}$ & $9(75)$ & $6(17)$ & $15 \quad(3.1-72)$ & 0.0004 \\
\hline $\begin{array}{l}\text { Caesarean section, in } \\
\text { labour }\end{array}$ & $1(8)$ & $6(17)$ & $0.5(0.1-4.2)$ & \\
\hline Pneumothorax & $5(42)$ & $9(25)$ & $2.1(0.5-8.5)$ & \\
\hline Hypotension & $11(92)$ & $28(78)$ & $3.1(0.4-28)$ & \\
\hline Patent ductus arteriosus & $5(42)$ & $11(31)$ & $1.6(0.4-6.3)$ & \\
\hline Sepsis & $8(67)$ & $11(31)$ & $4.6(1.1-18.3)$ & 0.04 \\
\hline Necrotising enterocolitis & $5(42)$ & $1(3)$ & $25(2.5-248)$ & 0.002 \\
\hline Neonatal seizures & $7(58)$ & $2(6)$ & $24 \quad(3.8-1.8)$ & 0.0003 \\
\hline
\end{tabular}

^ Fisher's exact test.

Table 6 Ultrasound findings expressed as number (\% of subjects scanned) in relation to presence and timing of ischaemic white matter damage

\begin{tabular}{|c|c|c|c|c|}
\hline \multirow[b]{2}{*}{ Variable } & \multicolumn{2}{|c|}{ Necropsy findings } & \multirow[b]{2}{*}{$\begin{array}{l}\text { Prenatal } \\
(n=7 / 12)\end{array}$} & \multirow[b]{2}{*}{$\begin{array}{l}\text { Postnatal } \\
(n=10 / 12)\end{array}$} \\
\hline & $\begin{array}{l}\text { No lesion } \\
(n=21 / 36)\end{array}$ & $\begin{array}{l}\text { All lesions } \\
(n=30 / 39)\end{array}$ & & \\
\hline Normal ultrasound findings & $7(33)$ & $2(7)$ & $0(0)$ & $1(10)$ \\
\hline $\begin{array}{l}\text { Maternal haemorrhage / } \\
\text { intraventricular } \\
\text { haemorrhage }\end{array}$ & $13(62)$ & $23(77)$ & $3(25)$ & $8(80)$ \\
\hline $\begin{array}{l}\text { Early }(0-9 \text { days }) \\
\text { parenchymal lesion }\end{array}$ & $1(5)$ & $17(57)$ & $5(71)$ & $3(30)$ \\
\hline $\begin{array}{l}\text { Late ( }>9 \text { days) } \\
\text { parenchymal lesion }\end{array}$ & $0(0)$ & $5(17)$ & $0(0)$ & $3(30)$ \\
\hline Hydrocephalus & $0(0)$ & $8(27)$ & $3(43)$ & $5(50)$ \\
\hline
\end{tabular}

* Parenchymal lesion: definite intraparenchymal haemorrhage/infarct or cyst lesion confirmed an at least two scans.

$5.0(1.2-21))$ and delivery without labour (odds ratio $15(3.1-72)$ ). Of the neonatal factors, sepsis (odds ratio $4.6(1.1-18)$ ), necrotising enterocolitis (odds ratio $25(2.5-248)$ ), and seizures (odds ratio $24(3.8-108)$ ) were associated with an increased risk of postnatal white matter damage.

Ultrasound scans were available for 21 $(58 \%)$ of the subjects with no lesions and 30 $(77 \%)$ of the subjects with ischaemic lesionsseven $(58 \%)$ prenatal subjects and $10(83 \%)$ postnatal subjects (table 6). The identification of lesions was reasonably consistent when ultrasound and necropsy findings were compared. Only one of the 21 cases without lesions appeared to have a parenchymal lesion on ultrasound which was not confirmed at necropsy. Of the 30 infants scanned who had ischaemic white matter at necropsy, 22 had parenchymal lesions, eight hydrocephalus, and two were normal. Five of the seven cases with prenatal white matter damage had lesions detected by ultrasound in the first nine days of life. Six of the 10 cases with postnatal white matter damage had lesions detected by ultrasound; three in the first nine days of life and three after the ninth day of life.

\section{Discussion}

There was neuropathological evidence of cerebral damage in $57 \%$ of babies examined; in
$83 \%$ these were white matter ischaemic lesions. In view of the site and characteristics of these lesions, it is plausible that, had they survived, they might have shown signs of cerebral palsy. This observation supports the overall view that the increasing rate and numbers of surviving very preterm babies with cerebral palsy reflects the survival of children with ischaemic white matter damage. ${ }^{1}$

Furthermore, in our study there was evidence that a third of the ischaemic lesions were of prenatal origin. This is consistent with findings in previous studies ${ }^{111415}$ and lends indirect support to the view that survivors who have sustained an in utero insult may contribute to the increased numbers of preterm babies with cerebral palsy.

Although the neuropathological examination clearly demonstrated prenatal ischaemic lesions, these were not associated with detectable antenatal clinical events. In previous studies clinical factors such as intrauterine growth retardation, oligohydramnios, and maternal infection have been associated with the development of prenatal ischaemic lesions. ${ }^{14-17} \mathrm{Al}-$ though adverse antenatal factors were common in the mothers of babies in this study, there were no clear differences between the prevalence of antenatal events in babies with and without prenatal lesions. The increased prevalence of delivery without labour among those with prenatal lesions may reflect an association with the underlying reasons for elective caesarean section, such as pre-eclampsia and intrauterine growth retardation. But the numbers were small and this finding could be attributed to chance.

It remains a possibility that prenatal ischaemic injury is triggered by factors which we did not consider, or which are not clinically measurable. This highlights the limited techniques available to us for determining fetal wellbeing and particularly cerebral function before delivery.

Although one third of babies had ischaemic lesions of prenatal origin, in a further third white matter damage seemed to have originated after birth. Among these, there was a clear association with adverse neonatal events such as sepsis, necrotising enterocolitis, and neonatal seizures. This association may be confounded by the later age at death among babies with postnatal lesions, allowing more time for these lesions to develop, but it is likely that major neonatal events, particularly if accompanied by hypotension and hypoxia, will contribute to cerebral ischaemia.

A more surprising finding was an association between postnatal lesions and adverse antenatal factors, including pre-eclampsia, intrauterine growth retardation, and delivery without labour (elective caesarean section). Recent work by us ${ }^{18}$ and by others ${ }^{19}$ has shown that in very preterm babies, pre-eclampsia is associated with a reduced risk of cerebral palsy. Kuban et al also demonstrated a reduced prevalence of intraventricular haemorrhage in the babies of mothers with pre-eclampsia. ${ }^{20}$ If the most severely affected preterm babies of mothers with pre-eclampsia die, this could 
account for a reduced risk of cerebral palsy among survivors. The role of pre-eclampsia and the effect of medications used in its management, such as magnesium sulphate, in cerebral ischaemia and cerebral palsy is unclear. ${ }^{18}{ }^{19}$ Randomised controlled trials of treatments for severe pre-eclampsia to test their effectiveness in reducing fetal and neonatal death, or cerebral palsy, or both among survivors will clarify these issues.

Meanwhile the observation that adverse antenatal events are associated with postnatal ischaemic lesions not only highlights the problem of relating the time of brain injury to the clinical findings, but also raises the possibility of a cascade of adverse events originating in the prenatal period and culminating in ischaemia in the brain and other organs after birth. Indeed, the view that cerebral ischaemic lesions are either prenatal or postnatal in origin may be rather simplistic. Biological studies suggest that the period in which the developing white matter is most vulnerable to hypoxicischaemic injury is from 26 to 34 weeks of gestation. ${ }^{21}$ Very preterm babies may be delivered at any point during this critical period and the effect of ischaemic damage needs to be related not so much to the event of birth as to the gestational age of the fetus or baby when an insult occurs. Perhaps we need to focus more on the response of an immature brain to a variety of insults which may occur at any point in this vulnerable period, and less on a polarised debate about the prenatal or postnatal origins of cerebral palsy. This theory is supported by experimental studies in fetal lambs where several short insults have been shown to produce more brain damage than one single insult of similar overall duration. ${ }^{22}$

Finally, there will always be concerns about the association between the ischaemic lesions seen at necropsy and those which are precursors of cerebral palsy in survivors. Advances in neuroimaging seem to offer the most promising way forward in identifying and timing the onset of cerebral ischaemic lesions in liveborn babies. Ultrasound scans detected parenchymal lesions in $73 \%$ of the babies with white matter damage at necropsy and the estimated time of onset of the lesions was consistent between the two investigations. At present, postnatal ultrasonography is the most widely used method of determining the presence and time of onset of these lesions in living babies. Newer methods of brain imaging such as magnetic resonance imaging (MRI) are now being introduced and may improve the sensitivity and specificity of detecting ischaemic lesions in the fetus and the newborn baby.

\section{Appendix 1}

DEFINITION OF ANTENATAL CHARACTERISTICS Antepartum haemorrhage: Vaginal bleeding after 20 weeks' gestation regardless of aetiology after exclusion of local haemorrhage of genital tract. Placental abruption: Severe haemorrhage (at least $200 \mathrm{ml}$ ) after 20 weeks' gestation with at least two of the following - a clinical diagnosis (painful haemorrhage with a tense uterus), evi- dence of substantial retroplacental clot at delivery, or histological evidence of abruption. Prolonged rupture of membranes: Rupture of the membranes for more than 24 hours.

Chorioamnionitis: Infection/inflammation of the membranes diagnosed by at least two of the following-clinical evidence (pyrexia, tender uterus, purulent liquor), microbiological evidence or histological evidence.

Maternal infection: Urinary tract infection or pyelonephritis (confirmed on mid stream urine), pyrexia ( $>38.5 \mathrm{C}$ ) of over 24 hours' duration, any other microbiologically confirmed infection other than chorioamnionitis. Pre-eclampsia: The presence of hypertension (diastolic blood pressure $\geqslant 90 \mathrm{~mm} \mathrm{Hg}$ ) and proteinuria (with or without biochemical and haematological changes).

Intrauterine growth retardation: Ultrasound derived diagnosis of a weight more than 2 standard deviations below the mean using John Radcliffe percentile charts, estimated by serial measurements of the abdominal circumference.

Small for gestational age: Birthweight more than 2 standard deviations below the mean as defined by percentile charts used at the John Radcliffe Hospital.

\section{Appendix 2}

DEFINITION OF POSTNATAL CHARACTERISTICS Hyaline membrane disease: Clinical diagnosis supported by chest $x$-ray and specifying whether surfactant was administered.

Pneumothorax: Clinical diagnosis supported by chest $x$-ray and requiring a chest drain.

Hypotension: Mean blood pressure $<30 \mathrm{~mm}$ $\mathrm{Hg}$ in the first 24 hours.

Patent ductus arteriosus treatment: Clinical diagnosis and specifying whether fluid restriction, indomethacin, or surgical ligation were required.

Sepsis: Clinical diagnosis supported by microbiological evidence of infection.

Necrotising enterocolitis: Clinical and radiological diagnosis, specifying whether surgery was required.

Neonatal seizures: Unifocal or multifocal fitting diagnosed clinically or with electroencephalography (EEG) and requiring anti-convulsant therapy.

We thank colleagues at the National Perinatal Epidemiology Unit for commenting on drafts of the paper.

DJ Murphy is funded by Action Research and A Johnson by the Department of Health.

1 Pharoah P, Cooke T, Cooke RW, Rosenbloom L. Birthweight specific trends in cerebral palsy. Arch Dis Child 1990;65:602-6

2 Leviton $\mathrm{A}$, Paneth $\mathrm{N}$. White matter damage in preterm newborns - an epidemiologic perspective. Early Hum Devel 1990;24:1-22.

3 Graham M, Trounce JQ, Levene MI, Rutter N. Prediction of cerebral palsy in very low birthweight infants: prospective ultrasound study. Lancet 1987;329:593-6.

4 Cooke RWI. Early and late cranial ultrasonographic appearances and outcome in very low birthweight infants. Arch Dis Child 1987;62:931-7.

5 Fawer CL, Diebold P, Calame A. Periventricular leucomalacia and neurodevelopmental outcome in preterm infants. Arch Dis Child 1987;62:30-6.

6 Weindling AM, Rochefort MJ, Calvert SA, Fok T, Wilkinson A. Development of cerebral palsy after ultrasonographic A. Development of cerebral palsy after ultrasonographic detection of periventricular cyst 
7 de Vries LS, Dubowitz V, Lary S, Whitelaw A, Dubowitz LMS, Kaiser A, et al. Predictive value of cranial ultrasound in the newborn: a reappraisal. Lancet 1985; 337: 137-40.

8 Scher MS, Belfar H, Martin J, Painter MJ. Destructive brain lesions of presumed fetal onset: antepartum causes of lesions of presumed fetal onset: antepartung
cerebral palsy. Pediatrics $1991 ; 88: 898-906$.

9 Bejar R, Wozniak P, Allard M, Benirschke K, Vaucher Y, Coen $R$, et al. Antenatal origin of neurologic damage in newborn infants. 1. Preterm infants. Am $\mathcal{F}$ Obstet Gynecol 1988; 159: 357-63.

10 Sinha SK, D'Souza SW, Rivlin E, Chiswick ML. Ischaemic brain lesions diagnosed at birth in preterm infants: clinical events and developmental outcome. Arch Dis Child 1990;65:1017-20.

11 Squier $M$, Keeling JW. The incidence of prenatal brain injury. Neuropathol Appl Neurobiol 1991;17:29-38.

12 Squier MV. Fetal and neonatal pathology. In: Keeling JW, ed. Acquired diseases of the nervous system. London: ed. Acquired diseases of the

13 OSIRIS Collaborative Group. Early versus delayed neonatal administration of a synthetic surfactant - the judgement of OSIRIS. Lancet 1992;340:1363-9.

14 Gaffiney G, Squier MV, Johnson A, Flavell V, Sellers S. Clinical associations of prenatal ischaemic white matter injury. Arch Dis Child 1994;70:F101-F6.
15 Ellis WG, Goetzman BW, Lindenberg JA. Neuropathologic documentation of prenatal brain damage. $A m \mathcal{F}$ Dis Child 1988;142:858-66.

16 Sims ME. Brain injury and intrauterine death. Am $f$ Obstet Gynecol 1985;151:721-3.

17 Gilles FH, Leviton A Dooling EC. The developing human brain: growth and epidemiologic neuropathology. Boston: Wrain: growth

18 Murphy DJ, Sellers S, MacKenzie IZ, Yudkin PL, Johnson AM. Case - control study of antenatal and intrapartum risk factors for cerebral palsy in very preterm singleton babies. Lancet 1995; 346: 1449-54.

19 Nelson KB, Grether JK. Can magnesium sulfate reduce the risk of cerebral palsy in very low birthweight infants? Pediatrics 1995;95:263-9.

20 Kuban KCK, Leviton A, Pagano M, Fenton T, Strassfeld R, Wolff M. Maternal toxemia is associated with reduced incidence of germinal matrix hemorrhage in premature babies. I Child Neurol 1992;7:70-6.

21 Hill A. Current concepts of hypoxic-ischemic cerebral injury in the term newborn. Pediatr Neurol 1992;7:317-25. Mallard EC, Williams CE, Gunn AJ, Gunning MI Mallard EC, Williams CE, Gunn AJ, Gunning MI,
Gluckman PD. Frequent episodes of brief ischaemia Gluckman PD. Frequent episodes of brief ischaemia
sensitize the fetal sheep brain to neuronal loss and induce striatal injury. Pediatr Res 1993; 33:61-5. 